Culture et histoire dans l'espace roman

4 | 2010

Les représentations du corps dans la litterature latinoaméricaine

\title{
Il était une fois le corps
}

\section{Christine Détrez}

\section{(2) OpenEdition \\ 1 Journals}

Édition électronique

URL : https://journals.openedition.org/cher/8619

DOI : $10.4000 /$ cher.8619

ISSN : 2803-5992

Éditeur

Presses universitaires de Strasbourg

\section{Édition imprimée}

Date de publication : 30 juin 2010

Pagination : 17-25

ISBN : 978-2-35410-007-0

ISSN : 1968-035X

Référence électronique

Christine Détrez, " Il était une fois le corps », reCHERches [En ligne], 4 | 2010, mis en ligne le 15

décembre 2021, consulté le 26 janvier 2022. URL : http://journals.openedition.org/cher/8619; DOI :

https://doi.org/10.4000/cher.8619

\section{(c) (i) (2)}

Ce(tte) œuvre est mise à disposition selon les termes de la Licence Creative Commons Attribution Pas d'Utilisation Commerciale - Partage dans les Mêmes Conditions 4.0 International. 


\title{
Il était une fois le corps
}

\author{
Christine Détrez \\ Sociologue, ENS - LSH de Lyon
}

$\mathrm{U}$

tiliser cette expression qui ouvre les contes peut aller contre le sens commun qui verrait dans le corps un objet biologique, le produit d'un héritage génétique. Cela va également à l'encontre de l'idée que la science ou la médecine seraient les seuls discours légitimes et légitimés pour parler du corps. Il nous semble au contraire que la sociologie, la littérature, la photographie, la danse... peuvent parler du corps également, et sont tout aussi légitimes à le faire, et cela non pas seulement en décoration ou en illustration: contre l'idée encore trop souvent répandue de domaines réservés, de chasses gardées, au contraire, leurs discours ont eux aussi une vérité à amener, dans un système où les discours se complètent sans s'exclure.

Le thème du corps en sociologie permet ainsi de façon très précieuse de réfléchir aux liens entre culture et nature, entre individu et société, individu et groupe, entre stéréotype et expression littéraire.

\section{Le poids des héritages}

La difficulté à penser «sociologiquement» ou «socialement» le corps peut certes être replacée dans la longue lignée de l'héritage philosophique occidental. Très rapidement, il est possible de distinguer deux grandes traditions philosophiques, et d'envisager la place que chacune accorde au corps. L'une, héritée de Platon, pose la séparation entre l'âme et le corps, dans une hiérarchie où le corps est l'instance méprisée. Il suffit de se rappeler du jeu de mots de Socrate entre séma (tombeau) et soma (corps): 
le corps est ainsi le tombeau de l'âme. L'autre tradition, plus inspirée de Aristote, postule l'association et le modelage de l'âme et du corps. La théorie des humeurs se fonde ainsi sur une association entre le biologique et le caractère, le médical et le comportemental. C'est ainsi toute une science, la physiognomonie, qui va s'attacher à lire le caractère des individus sur leurs visages ou leurs postures, tradition qui, si elle a aujourd'hui perdu ses lettres de noblesse scientifique, n'en continue pas moins d'influer sur la littérature, le cinéma, voire certains départements universitaires américains, comme la Communication Non Verbale (Détrez 2002), ou encore les conseillers de nos hommes et femmes politiques.

Toute une tradition associe dans la même logique la beauté et la valeur morale d'une personne. La Princesse de Clèves s'ouvre sur cette description emblématique: «Jamais cour n'a eu tant de belles personnes et d'hommes admirablement bien faits; et il semblait que la nature eût pris plaisir à placer ce qu'elle donne de plus beau dans les plus grandes princesses et les plus beaux princes». George Vigarello (2004) décline cette association entre valeur morale et beauté, notamment par un examen des parties du corps privilégiées par l'art... et la mode: au XVI ${ }^{\mathrm{e}}$ siècle, le visage domine, de façon presque religieuse, la beauté étant la manifestation des vertus morales. Le bas du corps disparaît sous des jupes quasi à l'horizontale. Le corset est là aussi pour contrôler ce qui est montré. À la femme la beauté, à l'homme la force, toujours en relation avec la théorie des tempéraments, la première étant «par nature» molle, tendre, faible, et le second, vigoureux. Il est à peine besoin de préciser que ces qualités ne sont pas équivalentes. La beauté féminine est faite pour réjouir l'homme ou le servir, puis, (courant XVIII') pour l'attirer et faire de beaux enfants. Elle n'est qu'une façon d'assigner la femme à sa seule place: l'intérieur, puisque toujours "par nature», elle n'a pas assez de force pour aller dans le monde, et dans l'espace public. Comme le souligne Rousseau: «les femmes ne sont pas faites pour courir, quand elles fuient, c'est pour être atteintes». De même, l'idéal esthétique de la cambrure du XIXe siècle a des raisons... médicales: selon une encyclopédie de la médecine (1861) «la conformation du bassin (chez l'homme) donne l'image de la force, alors que celui de la femme indique sa destination relative à l'accouchement».

Sauf contre-exemples fonctionnant avec l'éclat de l'exception, les princesses des contes sont aussi bonnes que belles. Ce dernier exemple révèle également que traditionnellement, à la beauté physique est associée également la valeur sociale: les vraies princesses sont celles qui ont le pied le 
plus petit, le doigt le plus fin, le dos le plus délicat. Les contes, qui n'étaient pas tous écrits pour des enfants, fonctionnent alors comme justification de l'ordre social. Le dernier livre de Nicolas Herpin, Le pouvoir des grands, montre de même qu'aujourd'hui encore, les grands ont un avantage sur les autres, en termes aussi bien professionnel que sentimental. Jean-François Amadieu, dans Le poids des apparences, décrit de même toute une série d'expériences, où la note donnée par un professeur, le jugement d'un tribunal, etc. varient selon l'aspect physique du sujet, ou de la photo épinglée au dossier.

Mais la représentation qui va dominer et devenir la représentation médicale légitime est celle du corps machine, du corps automate. Elle se développe à partir du XVII e siècle, siècle de Descartes puis tout au

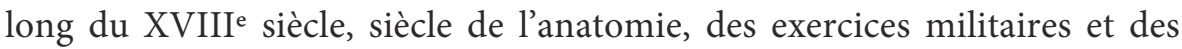
automates: Michel Foucault, dans Surveiller et punir, a bien mis en évidence l'importance de la figure de Frédéric II de Prusse, ce roi des machines et des exercices militaires... Une même discipline des corps se déploie alors dans la pratique de l'exercice militaire, dans les exercices de gymnastique, dans les salles de classe, les rangées d'hôpitaux, les rangs des armées, dans l'organisation du temps (les emplois du temps) et du savoir (avec la taxinomie et la classification): on quadrille, on sépare, on analyse, on met au pas et en examen... C'est ainsi un corps objectivable, découpable et analysable en éléments séparés comme autant de disciplines médicales, qui devient la norme.

Le corps est ainsi le nœud où s'articulent savoirs/ pouvoirs, et les exemples seraient légion. Un parmi d'autres, Malek Alloula, travaillant sur les cartes postales envoyées des colonies en métropole, décrit bien le processus «d'ethnographisation» de ces photos, où le classement en scènes et types de la population indigène est une façon parmi d'autres d'affirmer la supériorité et la maîtrise coloniales et occidentales.

\section{La construction sociale du corps}

Néanmoins, contre - ou en complément - de cette vision mécanique du corps, on peut poser que le corps varie selon les sociétés, historiquement, géographiquement, socialement: selon l'endroit de l'espace social où l'on est situé, selon l'identité sexuelle, selon le métier, le corps est formé, déformé, conformé. 
Les premières ruptures avec l'évidence biologique et l'essentialisation proviennent de l'anthropologie. Marcel Mauss, dans l'article célèbre «Les techniques du corps», part de ses lectures mais également de ses expériences personnelles - notamment pendant la guerre, occasion de côtoyer des hommes d'autres nationalités - pour montrer que le corps n'est pas «naturel»: ainsi, il se souvient ne pas avoir pu s'accroupir dans la boue comme les soldats australiens, ou il évoque l'incapacité des Anglais à se servir des bêches françaises, ou à marcher au pas comme les Français.

Une multitude d'études vont se déployer au tournant des années 40 avec le but explicite de "dénaturaliser le corps »: Gregory Bateson et Margaret Mead passent ainsi de longs mois dans la société balinaise, et en reviennent avec des milliers de photos et des kilomètres de films: ils observent par exemple que même au repos, alors que les contrôles «conscients» sont relâchés, les corps balinais ne sont pas comme les corps occidentaux, notamment les positions des mains, «tordues» selon les critères occidentaux.

Une autre enquête, dont on saisira la portée politique quand on saura qu'elle a été faite en 1942 par un juif émigré aux États-Unis vise de même à démontrer que le comportement des individus n'est pas racialement mais socialement façonné: David Efron, dans Gesture, Race and Culture, étudie ainsi deux générations italiennes et américaines pure souche. La comparaison entre la génération immigrée primo-arrivante et la génération américaine révèle effectivement des différences, dans la façon de se tenir, de se comporter, les premiers étant bien plus expressifs, bruyants etc. que les derniers. Mais la même comparaison menée sur des individus issus de la deuxième génération d'immigration montre bien davantage de différences entre ceux-ci et leurs parents qu'entre ceux-ci et les Américains "pure souche» : c'est donc bien la socialisation, et non pas la « race» qui modèle les corps, ses postures et attitudes.

Un des plus grands intérêts de l'anthropologie est sans nul doute de relativiser nos certitudes par la comparaison avec d'autres cultures. Le regard doit alors être tourné vers nos propres sociétés, afin de dénaturaliser les évidences. C'est ce que font également les statistiques, notamment lorsqu'elles détaillent l'évolution des morphologies sur plusieurs générations: l'enquête de Marceline Bodier montre ainsi que selon les générations, mais aussi, pour chaque génération, selon le groupe social, la morphologie évolue.Les Français seraient ainsi de plus en plus grands, les membres des classes favorisées étant plus grands que les autres. De même, la toute 
dernière enquête de mensurations a montré l'effacement de la taille chez les femmes, lié à l'évolution des modes de vie.

Des historiens ont montré de même l'évolution de ce qui pourrait passer comme une pure mécanique physico-chimique: les sens. Ainsi, Alain Corbin, dans Le Miasme et la jonquille, étudie comment s'élabore et se codifie socialement et historiquement la sensibilité aux odeurs: non seulement celles-ci diffèrent tout simplement selon les normes d'hygiène, mais les odeurs sont également porteuses de valeurs symboliques et sociales. Il associe ainsi le rejet au cours du XIXe des odeurs lourdes et capiteuses au profit des odeurs «de propre» à l'ascension de la bourgeoisie sur la noblesse. Les parfums y sont à la fois symboles d'oisiveté, et de dépenses ostentatoires et inutiles, associées à l'indolence contre les valeurs de travail et d'énergie portées par cette bourgeoisie. Par ailleurs, toujours selon Alain Corbin, les sens seraient organisés selon une hiérarchie: notre époque consacrerait ainsi la vue (on en trouve déjà les prémisses dans le panopticon de Bentham tel que le décrit Foucault), contre l'odorat. L'époque contemporaine serait ainsi le lieu d'un corps "présent-absent», qui ne doit se signaler ni par ses odeurs, ni par sa visibilité. Les corps perturbateurs rompent avec cette norme d'invisibilité: c'est le cas de ces corps trop manifestes que sont les corps obèses, les corps vieux, ou fous... qui rappellent que le corps est faillible, et l'homme mortel. Ici encore, le corps est porteur de valeurs symboliques et morales qui dépassent le simple relevé des mensurations. Dans une société de performance et de responsabilité individuelle, les corps «trop» gros, par exemple, encourent le stigmate et jugement moral: est gros celui qui est faible, qui n'a pas de volonté, et ne sait pas se maintenir, se contenir...

Si elle varie historiquement, cette sensibilité différenciée peut également être source de quiproquos culturels, entre individus de pays différents. C'est pour Edward T. Hall, selon le titre de son livre, une véritable «Dimension cachée». Selon les cultures, les individus ont ainsi une sensibilité différente par exemple aux bruits, et surtout à la "proxémie», c'est-à-dire à l'espace nécessaire à chacun pour ne pas se sentir agressé par la présence d'autrui, cette sorte de distance de protection, qui varie elle-même de façon codée selon les situations (la distance acceptable entre deux corps est ainsi différente, mais toute aussi normée, dans les transports en commun, les cours, la situation amoureuse, etc.).

Didier Fassin souligne ainsi le marquage des corps qu'opèrent nos sociétés occidentales contemporaines. Loin de ne voir dans ces marquages des corps que les peintures ou scarifications des sociétés traditionnelles, il 
faudrait alors lire dans ce modelage morphologique, physique et chimique de nos corps la formation, déformation et conformation spécifique de la vie en société, et ce de façon bien plus efficace que les peintures ou cicatrices traditionnelles, puisque ces formations / déformations / conformations sont incorporées, et non pas appliquées «seulement» de façon extérieure sur la peau. Ici encore, l'histoire de la mode est riche d'enseignements: quand la noblesse domine, les corsets projettent le buste en arrière. Au fur et à mesure de l'avènement de la bourgeoisie, le buste est au contraire propulsé en avant, par la redingote et le gilet. Chez les femmes, quand la robe se fluidifie à partir du XIX ${ }^{\mathrm{e}}$ siècle, le corset s'allonge pour enserrer et contrôler les parties visibles. Quand il disparaît, il se trouve en quelque sorte incorporé, puisque ce sont les muscles qui sont censés le remplacer...

C'est d'ailleurs un des principaux problèmes des campagnes de santé publique: les conseils et alertes qu'elles mènent sont surtout entendus par ceux qui, déjà, se soucient au préalable de leur santé. Si statistiquement, l'obésité, ou les maux dentaires par exemple, sont plus fréquents dans les classes moins favorisées, les conseils de nutrition, ou de suivi, ou de dépistage pour d'autres maladies, auront néanmoins plus d'effet sur les classes favorisées, et l'écart continue alors à se creuser...

En sus-même de la morphologie, la tenue du corps, l'hexis corporelle, montre comment la façon même de se comporter, de déplacer et bouger ce corps, est modelée par la place qu'on occupe: on n'apprend pas à un petit garçon et à une petite fille les mêmes gestes, on ne les encourage pas aux mêmes activités, on ne les forme pas à se tenir de la même manière en société. De la même façon, Pierre Bourdieu, dans des passages de la Distinction aux accents de Portraits de La Bruyère, dépeint les corps en action de la bourgeoisie, et des classes populaires, opposant réserve et ampleur, discrétion et bruit, etc.

Le corps varie donc selon les sociétés, et la place occupée dans chaque société, modelé par les valeurs morales, et sociales, attribuées à l'individu. En cela, le corps permet de penser l'articulation entre l'individu et la société, entre nature et culture. En effet, il se trouve au centre d'un double mouvement: la socialisation (variant selon la culture de chaque société) s'exerce sur un donné naturel. Cette socialisation s'applique de telle façon qu'elle s'incorpore, et devient ainsi une "seconde nature», s'imposant avec l'évidence du naturel, le naturel de l'évidence, à tel point que l'on pourra s'émerveiller de la "grâce naturelle» de quelqu'un, etc. Quelques exemples peuvent illustrer ces dialectiques entre nature et culture, individu et groupe: 
ainsi, un bébé doit atteindre une certaine maturité biologique et physique pour marcher. Quand il apprend à marcher, il le fait selon les exemples qui l'entourent. Mauss souligne ainsi combien les petites filles maori, par exemple, marchent de façon différente des petites filles françaises. On a alors la force du groupe, de la culture. Néanmoins, chaque individu a une démarche qui lui est propre, et qui le rend reconnaissable de loin... De la même façon, le sourire d'un bébé est une faculté physiologique; ce n'est qu'au fil des premiers mois qu'il apprend la signification sociale du sourire. Mais de même, chaque individu a son sourire, qui le distingue de son frère, de sa sœur, etc.

\section{Les mécanismes de cette incorporation du social:} socialisation, éducation et imitation

Ce sont souvent les situations problématiques qui soulignent, en creux, la force et l'invisibilité des socialisations réussies: c'est le cas par exemple des "garçons manqués», qui montrent, dans leur corps de petites filles ratées, car trop masculines, la force des socialisations sexuées. Dès la plus tendre enfance, voire avant, avec la connaissance du sexe du bébé, l'enfant apprend à être un corps de fille ou un corps de garçon, et à se tenir comme tel. Étudiant un corpus de photographies publicitaires, Erving Goffman a montré comment les poses des mannequins étaient «ritualisées»: mains devant la bouche cachant un sourire, pieds rentrés, attitudes frêles et fragiles... Certes, il s'agit de poses et de modèles, mais comme l'explique Goffman, celles-ci fonctionnent car elles s'inscrivent dans un réel codifié: la pose n'est ainsi qu'une «hyperritualisation» de la réalité.

De la même façon, les socialisations varient selon le milieu social: les romans autofictionnels d'Annie Ernaux, qui par sa réussite scolaire, devient une transfuge sociale, mettent bien en scène les situations de décalage entre la tenue du corps populaire, et le dressage de ce même corps par l'école. Les romans d'écrivains entre deux cultures - soit parce que les parents forment un couple mixte, soit par la marque de la colonisation sur l'école - dessinent aussi des corps socialisés: Assia Djebar évoque la langue française comme libératrice de son corps, qui apprend à courir et à sauter, mais ne peut plus s'accroupir avec les autres femmes. Georges Vigarello (1978) l'a bien montré, une des missions de l'école était de dresser les corps et les langages populaires, de faire de ces élèves populaires des individus "polis » à tous les sens du terme. 
Cette socialisation corporelle n'est pas limitée à l'enfance: elle continue tout au long de la vie: les sports, les métiers forment et déforment les corps. Il suffit de voir, pour s'en convaincre, les pieds des danseuses classiques, ou les corps des nageurs... Ainsi Sylvia Faure rapporte les témoignages de danseuses classiques passant à la danse contemporaine, et observant les modifications de leur morphologie... Rappelons également qu'il est possible, pour les archéologues, de deviner le métier des individus en analysant certaines déformations de leur squelette.

La socialisation corporelle, cette incorporation du jeu social s'effectue de deux principales façons, définies par Marcel Mauss dans son article «Les techniques du corps»: l'éducation (les remarques explicites, les «tiens-toi droit» par exemple, dont la répétition est vouée à disparaitre, une fois que l'injonction a été intériorisée) et l'imitation. C'est par ce dernier point que Mauss explique les démarches des petites filles maori, qui marchent comme elles le voient faire par leur mère.

\section{Jeux et enjeux de pouvoir}

Le corps est, nous l'avons vu, socialisé, modelé par la place occupée sur l'échiquier social. Mais le corps peut devenir jeu et enjeu de pouvoirs, de dominations concrètes et symboliques, qu'il s'agisse de dominations sociales ou genrées.

Au sens propre, le corps peut être objet marchand. C'est le cas bien évidemment de la prostitution, voire du trafic d'organes, du «don» du sang aux États-Unis (qui n'a de don que le nom, puisqu'il est rémunéré) ou des marchés des mères porteuses: dans tous ses cas, ce sont les corps des pauvres qui servent aux riches, qu'il s'agisse de classes sociales, ou de continents (Actes de la recherche en sciences sociales).

Autre domination bien concrète, les violences sur les femmes, qu'elles soient organisées au niveau national (que l'on songe au déficit de naissances féminines - le peuple manquant - en Chine ou en Inde), ou au niveau familial (l'enquête Enveff a ainsi fait grand bruit en France, en révélant les statistiques de femmes ayant déclaré avoir subi des violences).

Mais les dominations corporelles peuvent être également symboliques. Ainsi, Pierre Bourdieu a bien montré combien la domination sociale passe aussi par l'imposition des modèles de la classe favorisée: c'est le cas de la culture scolaire, mais également, dans le domaine corporel, de représentations du corps idéal. Or, ce corps idéal est toujours celui de la classe 
supérieure: corps blanc quand le bronzage trahit le travail, corps hâlé quand, quelques siècles plus tard, le bronzage est synonyme d'accès aux vacances et au soleil. La silhouette est également révélatrice: corps plantureux quand il montre le niveau économique, corps mince et musclé quand se révèle ainsi la possibilité de consommer une alimentation équilibrée, et d'entretenir son corps par le sport, ou quand il devient synonyme et preuve de responsabilité de soi, de prise en main. Les normes de la libération corporelle seraient ainsi les derniers avatars de cette imposition de modèles implicites: le corps libre de s'exhiber est justement ce corps mince, musclé et jeune des classes favorisées, c'est-à-dire un corps "performant».

La perspective du genre est bien évidemment primordiale pour l'analyse du corps, sans oublier que les normes et stéréotypes touchent aussi bien les femmes (avec la «féminité»), que les hommes (avec la «virilité»)... La domination genrée passe également par l'ordre symbolique. Ainsi, Françoise Héritier définit "la valence différentielle des sexes». Elle repère dans toute société des couples dichotomiques: gauche/droite, sec/mouillé, chaud/froid, haut/ bas... homme/ femme. Dans ces systèmes dualistes, les associations varient: dans telle société, l'homme sera associé au haut et la femme au bas, dans telle autre, ce sera l'inverse. L'invariant est néanmoins que, quelle que soit la société, la femme est toujours associée au terme hiérarchiquement inférieur de la dichotomie. Dans les systèmes symboliques, les différences sociales entre hommes et femmes se trouvent fondées sur des explications biologiques, des essentialisations naturelles, hormonales et corporelles. Ainsi, un travail (Détrez 2006) mené sur les encyclopédies traitant du corps destiné aux enfants montre que les corps de petites filles servent à expliquer les hormones, les réflexes (l'involontaire), et les corps de petits garçons les musclent et... le cerveau (le volontaire). Et il ne s'agit pas là de science expliquée aux enfants: des études menées sur les manuels de médecine, ainsi que sur certains des présupposés de la neurobiologie (Martin, BenoîtBrowaeys, Vidal) mettent en exergue les hypothèses particulièrement sexistes sous-tendant les expériences, qui aboutissent ainsi à naturaliser biologiquement des différences sociales.

Il est ainsi intéressant en conclusion de revenir sur les différences entre le discours que porte la sociologie sur le corps et les discours des siècles précédents, qui stipulaient notamment que les caractères (moraux) façonnaient les corps, et reflétaient la valeur sociale. Il semblerait au premier abord que la sociologie ne dise pas grand-chose d'autre: ce serait en effet les caractéristiques sociales qui façonneraient les corps. Mais les discours des 
siècles précédents tendaient à justifier l'ordre établi. La sociologie quant à elle, quand elle parle du corps, vise - entre autre - à révéler les rapports de domination qui se tissent et font des corps des jeux et enjeux de pouvoirs, concrets et symboliques.

\section{Conclusion: Et la littérature dans tout ça?}

Nul hasard dès lors si l'apparition des femmes sur la scène artistique, qu'il s'agisse de littérature, de cinéma, d'art contemporain ou de danse, est effectuée sous les auspices de l'exposition et de la revendication de leur corps. N'était-ce pas d'ailleurs notamment par «l'écriture du corps» que, pour les féministes des années soixante-dix, allait passer la libération de la parole féminine?

$\mathrm{Ne}$ voir dans les représentations artistiques qu'un épiphénomène reviendrait en effet à méconnaître la force symbolique des représentations, et le lien inextricable entre imaginaire et réalité. Par ailleurs, ne les considérer que comme des "reflets", plus ou moins déformés par la subjectivité de chaque artiste, d'une réalité extérieure et objective, serait négliger leur rôle dans la constitution comme dans le renouvellement de celle-ci. Les œuvres de fiction, quel qu'en soit le support, peuvent ainsi être envisagées comme le lieu d'un démarquage par rapport aux représentations dominantes tout autant que comme des documents qui les expriment. Il n'en reste pas moins que, par le jeu de la grande diffusion et de la médiatisation, les œuvres agissent sur les représentations et les façonnent...

Le corps, une histoire de bonnes femmes? Au contraire: l'expression de l'intime et la représentation du corporel, par les discours, les images ou les gestes, loin d'être coupée du réel et du social, est au contraire un acte éminemment politique - qui concerne la cité, au sens le plus plein du terme.

\section{Bibliographie:}

Actes de la recherche en sciences sociales, «Le commerce des corps», 1994, nº 104. Alloula, Malek, 2001, Le harem colonial, Paris, Séguier.

Amadieu, Jean-François, 2002, Le poids des apparences. Beauté, amour et gloire, Paris, Odile Jacob.

Bateson, Gregory, et Mead, Margaret, Balinese Character: a Photographic Analysis, 1942, New York, Academy of Science. 
Benoit-Browaeys, Dorothée et Vidal, Catherine, 2005, Cerveau, Sexe et Pouvoir, Paris, Belin.

Bodier, Marceline, «Le corps change, son image aussi», 1995, Insee Première, n 356.

Bourdieu Pierre, 1979, La distinction, Paris, Éditions de Minuit.

Corbin, Alain, Le miasme et le jonquille, 1982, Paris, Aubier.

Détrez, Christine, 2002, La construction sociale du corps, Paris, Le Seuil.

Détrez, Christine, 2006, «Il était une fois... le corps. La construction biologique du corps dans les encyclopédies pour enfants", Sociétés contemporaines, $\mathrm{n}^{\circ}$ 59-60, p. 161-175.

Efron, David, Gesture, Race and Culture, 1942, La Hague-Paris, Mouton.

Fassin, Didier, 1996, L'espace politique de la santé. Essai de généalogie, Paris, PUF.

Faure, Sylvia, 2000, Apprendre par corps, Paris, La Dispute.

Foucault, Michel, Surveiller et punir, 1975, Paris, Gallimard.

Goffman Erving, 1977, "La ritualisation de la féminité», Actes de la Recherches en Sciences Sociales, $\mathrm{n}^{\circ} 14$.

Hall, Edward T., 1971, La dimension cachée, Paris, Seuil.

Héritier, Françoise, 1996, Masculin, Féminin: la pensée de la différence, Paris, Odile Jacob.

Herpin, Nicolas, 2006, Le pouvoir des grands, Paris, La Découverte.

Martin, Emily, 2001, The Woman in the Body, Boston, Beacon Press.

Mauss, Marcel, «Les Techniques du corps», Sociologie et anthropologie, 1980, Paris, PUF.

Synesthésie, "Contemporaines. Genre et représentation», n²12: http://www. synesthesie.com/syn12/index.html.

Vigarello, Georges, 1978, Le corps redressé, Paris, J. P. Delarge.

Vigarello, Georges, 2004, Histoire de la beauté, Paris, Seuil. 